\title{
An Exploration of Black, Asian and Minority Ethnic Women's Perceptions of Public Health Messages to Reduce Stillbirth in the UK: a Qualitative Study.
}

Tomasina Stacey ( $\sim$ t.stacey@hud.ac.uk)

University of Huddersfield

Melanie Haith-Cooper

University of Bradford

Nisa Almas

University of Bradford

Charlotte Kenyon

University of Huddersfield

\section{Research Article}

Keywords: Pregnancy, public health, stillbirth prevention messages, Black, Asian and Minority Ethnic women

Posted Date: January 20th, 2021

DOl: https://doi.org/10.21203/rs.3.rs-146922/v1

License: (c) (i) This work is licensed under a Creative Commons Attribution 4.0 International License. Read Full License 


\section{Title page}

2 An exploration of Black, Asian and Minority ethnic women's perceptions of

3 public health messages to reduce stillbirth in the UK: a qualitative study.

4

\section{Authors}

$6 \quad *$ Tomasina Stacey ${ }^{1,2}$

$7 \quad$ Melanie Haith-Cooper ${ }^{3}$

$8 \quad$ Nisa Almas ${ }^{3}$

9 Charlotte Kenyon ${ }^{2}$

10

1. School of Human and Health Sciences, University of Huddersfield, Huddersfield, United

12 Kingdom

2. Calderdale and Huddersfield NHS Foundation Trust, Lindley, Huddersfield, United Kingdom

3. Faculty of Heath Studies, University of Bradford, Bradford, United Kingdom

* Corresponding Author

17

t.stacey@hud.ac.uk

Ramsden Building R1/20

19

Department of Nursing and Midwifery 
Abstract

Background: Stillbirth is a global public health priority. Within the United Kingdom, perinatal mortality disproportionately impacts Black, Asian and Minority Ethic (BAME) women, these communities have double the rates of stillbirth than white women. Although the explanation for this remains unclear, it is thought to be multidimensional and includes access to key public health information. Improving perinatal mortality is reliant upon raising awareness of stillbirth and its associated risk factors. The aim of this study was to explore BAME women's awareness of stillbirth, perceptions of how it can be prevented through maternal behaviour change and how key public health messages could be more accessible to BAME women.

Method: Two semi-structured focus groups and 13 one to one interviews were completed with a purposive sample of 30 participants from 18 countries and across 4 NHS Trusts. Participants provided a detailed account of their recollection of stillbirth advice and risk factors both before and during pregnancy. They also suggested approaches to more effectively communicate key messages to BAME women.

Results: The results indicate limited awareness of stillbirth and potentially modifiable risk factors. They highlight the importance of the way in which resources to communicate key messages are developed and how they are communicated. The study supports the need for a range of resources which should be developed and shared within communities and as well as by trusted health professionals.

Conclusion: Our study found that the term "stillbirth" does not routinely exist in many women's narrative of perinatal health. This introduces additional complexity for health professionals when discussing stillbirth prevention with women from culturally and linguistically diverse backgrounds. Through co-development messages can be more accessible, acceptable and communicated effectively. It was clear that there was no 'one size fits all' and that a multi-pronged approach is required.

\section{Keywords}

Pregnancy; public health; stillbirth prevention messages; Black, Asian and Minority Ethnic women 
54

Perinatal mortality is a global public health issue and there are wide variations in the rates of stillbirth across the world, even between and within high income countries (1). For the last 5 years, reducing stillbirth has been a national priority within the United Kingdom (UK) and there has been a $16 \%$ reduction from 4.2 stillbirths per 1000 total births in 2013 to 3.5 per 1000 in 2018 (2). However there remains a considerable disparity in outcome within the population, with Black, Asian and Minority Ethic (BAME) women continuing to have higher rates of stillbirth than white women (2). The reasons for these inequalities in stillbirth rates are not clear, but are likely to be multifaceted and include underlying socio-economic disadvantage and associated stress and coping mechanisms, access and acceptability of health care and accessibility to key public health messages around reducing stillbirth risk (3, 4).

Raising awareness of public health issues and associated risk factors is an important element in the reduction of poor health outcomes (5). A number of potentially modifiable risk factors for stillbirth have been identified in the last few years. These factors include, but are not limited to: optimal body mass index prior to conception (6), smoking cessation in pregnancy (7), screening for gestational diabetes (8), awareness of reduced fetal movements (9), maternal side going-to-sleep position in pregnancy (10), and reducing caffeine intake in pregnancy (11). In order for women to make positive behaviour choices they need to be aware of what factors are of importance. However, it is unclear whether key health messages relating to these factors are available, accessible and appropriate for diverse communities within the UK, especially women who may experience language and cultural barriers when accessing maternity care. This study aimed to explore BAME women's awareness of stillbirth, 
their perceptions of how stillbirth can be prevented through maternal behaviour change and how key messages could be more effectively communicated to BAME women.

\section{Methods}

Ethical approval was gained from the University ethics committee SREIC/2019/132 and a qualitative study was undertaken between November 2019 and May 2020. After acquiring informed consent, women were recruited to the study through "gatekeepers" at local voluntary sector organisations. Women were eligible to participate if they were from a BAME community, had migrated to the UK at some point in their lives, were aged over 18 , had a child under the age of five born in the UK and spoke either English or a language where a voluntary sector interpreter could be arranged. Women who had previously experienced an adverse outcome were excluded from the study. The gatekeepers were provided with study information, interested women were then invited by the research team to attend a face to face group or individual telephone briefing session to discuss the study further. Purposive sampling was undertaken to ensure the sample included women from various countries of origin, different cultural backgrounds and with different levels of English language literacy. Before the COVID-19 pandemic, two focus groups of women were conducted. During the COVID-19 pandemic, interviews were arranged on a one to one basis over the telephone. The same semi-structured interview guide was used for the focus groups and interviews. The questions related to knowledge of stillbirth prior to pregnancy, who communicated the key messages around reducing stillbirth risk in pregnancy and what these messages were. Further questions explored what participants believed would be the best way to communicate such messages in the future. Probes were used to explore the issues in more detail. See Supplementary table 1 for the interview guide. 
Interviews were audio recorded, transcribed verbatim and thematic analysis was conducted, using Word and the highlighting functions, following the principles from Braun and Clarke (12). Two members of the team (MC and TS) read all the transcripts, searching for patterns in the data to develop codes which were then used to construct themes. Other members of the team also read the transcripts and checked the analysis process. Discussion took place until consensus was reached on the final themes.

\section{Results}

In total, 30 women took part in the study, seventeen took part in two face to face focus groups and a further thirteen took part individual interviews via phone due to the COVID-19 pandemic, they had given birth in one of four North of England Trusts (see table 1 for full details).

\begin{tabular}{|c|c|c|c|c|c|}
\hline Participant & Type of interview & Home country & Time in UK & Age & Parity \\
\hline 1. $\mathrm{FG} 1$ & Focus group & Pakistan & 8 years & 34 & P2 \\
\hline 2. $\mathrm{FG1}$ & Focus group & Pakistan & 2 years & 30 & P1 \\
\hline 3. $\mathrm{FG1}$ & Focus group & Pakistan & 5 years & 23 & $\mathrm{P} 2$ \\
\hline 4. FG1 & Focus group & Pakistan & 3.5 years & 24 & P1 \\
\hline 5. $\mathrm{FG1}$ & Focus group & Bangladesh & 1 year & 26 & P1 \\
\hline 6. $\mathrm{FG1}$ & Focus group & Pakistan & 1 year & 27 & P1 \\
\hline 7. FG1 & Focus group & Hong Kong & 2.5 years & 24 & P1 \\
\hline 8. FG1 & Focus group & Pakistan & 1.5 years & 27 & $\mathrm{P} 2$ \\
\hline 9. $\mathrm{FG1}$ & Focus group & Pakistan & 2 years & 25 & P5 \\
\hline
\end{tabular}




\begin{tabular}{|c|c|c|c|c|c|}
\hline 10. FG2 & Focus group & Ethiopia & 7 years & 32 & P4 \\
\hline 11. FG2 & Focus group & Not stated & 3 years & 39 & P4 \\
\hline 12. FG2 & Focus group & Guinea & 1 year & 30 & $\mathrm{P} 2$ \\
\hline 13. FG2 & Focus group & Somalia & 5 years & 40 & P7 \\
\hline 14. FG2 & Focus group & Tunisia & 10 years & 32 & $\mathrm{P} 2$ \\
\hline 15. FG2 & Focus group & Congo & 3 years & 26 & P3 \\
\hline 16. FG2 & Focus group & Congo & 2 years & 30 & $\mathrm{P} 2$ \\
\hline 17. FG2 & Focus group & Congo & 2.5 years & 30 & $\mathrm{P} 2$ \\
\hline 18. & $\begin{array}{l}\text { Individual telephone } \\
\text { (with interpreter) }\end{array}$ & Saudi Arabia & 6 years & 36 & P1 \\
\hline 19. & Individual telephone & Egypt & 2.5 years & 38 & $\mathrm{P} 4$ \\
\hline 20. & Individual telephone & Yemen & 2 years & 32 & P1 \\
\hline 21. & Individual telephone & Pakistan & 4 years & 32 & P1 \\
\hline 22. & Individual telephone & Russia & 19 years & 30 & P3 \\
\hline 23. & Individual telephone & Albania & 1.5 years & 24 & P1 \\
\hline 24. & Individual telephone & Sudan & 3 years & 29 & P1 \\
\hline 25. & Individual telephone & Ethiopia & 11 years & 31 & P1 \\
\hline 26. & Individual telephone & Iran & 6 years & 36 & $\mathrm{P} 2$ \\
\hline 27. & Individual telephone & Uganda & 5 years & 22 & P1 \\
\hline 28. & Individual telephone & Senegal & 9 years & 33 & P1 \\
\hline 29. & Individual telephone & Sudan & 10 years & 31 & P3 \\
\hline 30. & Individual telephone & Eritrea & 3 years & 28 & P1 \\
\hline
\end{tabular}


112 Four main themes emerged from the data: limited awareness of stillbirth and associated risk

113 factors, mixed messages, the importance of the health professional, multiple information

114 sources.

\section{Stillbirth awareness}

116

117

Women were asked what they understood by the word stillbirth. Most women had never heard the word in English and for some the word stillbirth did not exist, or they had not come across it, in their first language:

'Naturally die, no I never heard about it, no, I'm not sure if we have any word in my language about it, no.' P26 Iran

Some women discussed how stillbirth may be described in their first language:

'when the baby born and he's dead, they just say the baby's dead, they don't have like a word like the stillbirth' P22 Russia

Some women discussed how a baby dying in pregnancy was a taboo subject in their culture which led to a lack of discussion or sharing of information:

'When the neighbours used to come around and I would hear that there's been a death but we never found out what happened, how it happened just they used to say Oh baby died in the stomach.' P21 Pakistan

Other women talked about how stillbirth was so common back home that it was openly discussed but there was a lack of understanding of the reasons why it happened 'in Ethiopia so many, yeah, child die, people like children in pregnancy times they don't know, that's why so many children die in pregnancy time' P25, Ethiopia 
Interestingly although women did not understand the term stillbirth and believe they never discussed it in pregnancy when questioned about specific advice they may have received they were knowledgeable about some preventative measures, but not why. 'she used to ask things like is the baby moving full stop... No nobody told me why this question was being asked' P21 Pakistan

'I don't think I remember anything about while I was pregnant to prevent child stillbirth... the ninth month and I was told maybe sleep onto your side just because you can breathe better ...but it wasn't about the child.' P20, Yemen

Many women discussed how the topic of stillbirth can be frightening and how providing key messages to reduce BAME women's risk of stillbirth could increase stress levels in women who may already be living difficult lives. They believed it was important for health professionals to frame information in a positive way to avoid this:

'I think it's best that you do not mention stillbirth...to a woman who is pregnant (antenatal) review. I have seen both sides ladies are pregnant and have problems at home thinking all the time ...this might happen to me or it might not happen to me. I think it's better if we give the lady advice about taking care of herself that is better for her. we should ask women about the movement of the baby and give them information about sleeping on your side and don't sleep on your back. Not use the word stillbirth' P 28, Senegal

In addition, some women discussed the idea of fate and that stillbirth will just happen with no underlying reason therefore mentioning it would cause stress in women unnecessarily: 
'I mean if it happened it happened...you won't need to hear about it...just thinking all your pregnancy...scared and stressed..... most of the ladies they say there is no reason for that.' P24, Sudan

\section{Mixed messages}

None of the women reported receiving specific advice from either health professionals or family and friends to reduce their risks of stillbirth. However, many of the women discussed how they received advice about keeping themselves and their unborn baby healthy. Advice received from family or friends, including in their home country, was often in conflict with advice received by health professionals in the UK. This included dietary advice:

'during the first three months they said I don't have to eat some things like banana...the food that is warm, especially they mention banana or date and then after three months yes again because it's boy I have to be careful about my diet, I'm not allowed to eat fish and just salmon, and seabass...' P26, Iran

'So I think the midwife would say "you're only allowed so much tuna a week" and then people in Russia would be like "oh I don't know what you're talking about, you can eat as much of this as you want...So it's just little things like that that would get confusing' P22, Russia

Women also received conflicting advice from different family and friends back home. In one case, a woman from Eritrea was advised to stay active by her family '...they told me you have to work to work, work, work because it's good for you, don't stay at home...' P30 Eritrea

She was also advised not to stay active by members of her church: 

'when I go, they said sit here, don't stand up a lot, not too much, too long time, you have to sit here, they told me like that, rest, like don't stand in our church...' P30 Eritrea

Advice offered included other aspects of daily life:

'don't even think about having a nice relaxing bath, whereas here the midwives are always like "enjoy your nice bubble bath", I mean obviously not scolding hot but you can have a normal bath with bubbles, you don't need to be worried about putting in oils and stuff, whereas yeah, in Russia be like "just be careful, be very careful"...' P22 Russia

As well as mixed messages about keeping healthy in pregnancy, women recalled advice received from midwives and antenatal classes that was inaccurate. This particularly related to the urgency of searching for support if there was a perception of decreased fetal movements, or how to stimulate movements:

'Yes, she asked me if I don't feel any movement for two days I think, if I remember, two days I have to go to the hospital' P26, Iran

'And then sometimes I'm calling for midwife and then they told me eat like ice cream, like ice, you need to cold the baby and then they move.' P25, Ethiopia

\section{The importance of the health professionals}

All the women discussed how they received advice from their midwife in pregnancy around staying healthy, although they could not always link the advice to reducing the risk of stillbirth. Women believed that information targeting BAME women to reduce their risk of stillbirth should be primarily communicated through health professionals, in particular midwives. Midwives, nurses, doctors, GPs and health visitors were all named as key people 
with this responsibility. It was believed that the information from health professionals was the most trustworthy when compared to other sources:

'...the midwives maybe should advise more the clients, the patients, because at least in my culture, in my country, when a doctor says something or when a nurse says something, when a midwife, it's more trustful. And the patient take it more seriously than they will take in information on internet or in a leaflet...' P23, Albania

In addition, women believed that the key messages around stillbirth prevention for BAME women should be ongoing throughout pregnancy to avoid women turning to less reliable sources of information:

'The best way is update the information... at least once a month because sometimes new mothers, they don't know anything, they take any advice from anyone, maybe that advice is not suitable for them, you have to go to midwife, doctor is better than internet.' P29, Sudan

Women were asked about the practicalities of receiving the key messages in terms of location and timing. Most believed they should be communicated in the woman's home, hospital or GP surgery and it was important that women were aware about reducing the risk of stillbirth pre-conceptually as well as throughout pregnancy:

'Well, I think maybe it should be before. Sometimes some ladies, before they get pregnant they have use with drugs and alcohol, and that's why it can make their baby die. So maybe it's better to start before' P23, Albania

Some women discussed how cultural barriers reduced the likelihood of BAME women attending for routine appointments and this needed to be addressed as a key element of stillbirth reduction strategies: 
'I think first of all we should advise them to go see a hospital...African communities, most of them, they don't go to hospital because some of them, they give birth at home, to get checked.' P28, Senegal

Trust was considered an important issue for most women, especially those who did not have family and friends in the UK and women believed it was the responsibility of the midwife to sources outside of maternity services and that this approach could be useful to supplement 
perspectives of these different sources varied between women. Some women discussed

how they relied on other sources to corroborate the message provided by the health professional:

'...my friends here from Iran, they always check internet, while the midwife told them, or the family told them, but they always check the website, the same as me...' FG2

In focus group two, the discussion revolved around the cultural background of the midwife compared to the woman:

'because my midwife was from Pakistan, and okay so from another culture, another experience and so I check the internet...' FG2

Some women discussed how written information would be useful to communicate key messages about reducing stillbirth:

'Yeah, a leaflet and give to, give it to pregnant women when they have got appointment with midwives and it's simple and explained to make sure they understand' P24, Sudan

However, other women did not think written information was very helpful. Many did not, or could not, read the information provided by health professionals and would not read the key messages around reducing stillbirth:

'Being told verbally is more effective than having the information because sometimes people don't read...or can't read...or can read but can't understand' FG1

In addition, one woman talked about how some BAME pregnant women may have a stressful life which is not conducive with receiving written information: 

of like things going on in your body, so people are weak, people are tired every time. I don't think leaflets would be a good idea to just sit down and read.' P27, Uganda

267

Many of the women used the internet to source information related to pregnancy. This related to times when there was no midwifery contact:

'Well the first three months I didn't have much information, just because I didn't see my midwife and I was just Googling most of the things'.. P19 Egypt

In particular the NHS site was a popular place to check. However, this was thought to be too general for some of the information women required.

Most women believed that creating a video would be an effective way of communicating key messages around stillbirth prevention:

'everybody likes to watch a video, especially if it's short and snappy because if it starts, it if becomes like a documentary a lot of people switch off... 'P22, Russia

It was believed that whatever way technology was used, it should complement the advice given by the health professional:

'And the midwife just talk about it and explain it and then the people can go home and the information is all the time with them, and they can check'. P26, Iran

It was also thought that a video would help women understand the message: 'films with graphics and things, that you can see not only hear, it might be a good way to spread that information, just because if you don't understand what's been saying you can have the photos or pictures and you can understand it'. P21, Pakistan 
Some women discussed how they had used mobile applications in pregnancy for general advice. They suggested the idea of the key messages about stillbirth prevention being provided on a mobile application downloaded onto a woman's smartphone: 'Yeah, actually I got some information from an app.' FG2

However, two women identified that they did not have regular access to the internet through a smartphone during their pregnancies, and it was felt that some women would need instruction on how to use an app:

'Some people they don't know how to use, not everybody' FG2

Some women thought text messages could be used as a reminder about the message.

However, other women thought that this may not be good for some women:

'...some women maybe they will not care about the message you know, because it's a message sometimes they delete it...' P29, Sudan

Social media was suggested by some women as a place to gain information about stillbirth, although other women discussed how accessing social media in relation to pregnancy could be frightening:

'...so I've been through many reviews, people they say, they speak about the experience and they said, look, I'm about to give birth and the baby is dead, so it was very bad, things to read, you know... so it's a bit scary...Fg2

A number of women discussed how they found it helpful to receive information about pregnancy in a group setting with peers and that this could be effective in communicating key messages about stillbirth prevention: 

'there is a class I used to go to... where...pregnant women...came together and they taught us ... when the baby comes how you carry them or what you have to do...like even how you sleep, how you do all that. So those classes I think really helped because... we were all involved...people from, or maybe from other countries who don't understand English very well, so as long as someone is standing there and practising it or showing them what to do, they kind of get the idea...' P27, Uganda

However, it was acknowledged that not all women had the opportunity to attend such groups.

Some women felt that information could be provided in an existing community group setting, with different agendas designed for women. Ideas included women's groups in mosques but also other opportunities:

'Like we have ESOL classes and there's speakers coming in, so there can be a talk about stillbirth.' FG1

It was felt that existing relationships between women in community groups would be beneficial when receiving advice about stillbirth prevention:

'I like to know people, to meet people and have a good relationship with them. Especially those, they will be, you know, like me, preparing for pregnancy.' P20, Yemen

Women who were living in large accommodation centres for new arrivals felt that these provided an opportunity to target a group of women to discuss stillbirth prevention: 'refugees and asylum seekers you could find them in the homes where they firstly come... and they keep them there for $3 / 4$ weeks' P20, Yemen

\section{Discussion}


This study found that, in general, there was limited awareness of stillbirth and the potentially modifiable factors that might be associated with it amongst women from culturally and linguistically diverse communities in the UK. Our findings have provided an insight into the variety of ways in which women receive information about keeping their babies safe in pregnancy and the mixed (and sometimes inaccurate) messages that they receive, even from health professionals themselves. We found that women have individual preferences for how stillbirth reduction messages would be best communicated including written information, use of the internet, social media and learning in group contexts. However, women believed that the health professional, in particular the midwife, is key in communicating these messages which other media can support. Our study highlights the importance of both the way in which communicated. In particular, the provision of a range of resources developed and shared within communities and supported by trusted health professionals.

Although there has been a considerable increase in focus on stillbirth prevention during the past decade, both nationally and internationally, there remains a low level of public awareness of stillbirth and the associated risk factors (13) and a continued stigmatisation of the condition . (14) (15),. Our findings reflect this lack of awareness and stigma in the fact that despite some BAME women having heard simple descriptions describing the physical outcome ("death of baby in stomach") they did not consider this to be indicative of a health issue or have a term to describe the specific mortality. A lack of acknowledgment that pregnancy complications exist is a real barrier to accepting public health messages to improve 
outcomes $(16,17)$. Bringing stillbirth out of the shadow of stigma and taboo is an important first step towards effectively communicating key messages to prevent stillbirth.

352 In order for women to alter their behaviour in response to public health messages, these messages need to be accessible and acceptable and they need be communicated effectively. As our study shows, who and how information is developed and shared is significant. One of the aims of our study was to understand how key messages could be more effectively communicated to BAME women and we found that women preferred a range of resources available in different formats. It was clear that there was no 'one size fits all' and that a multipronged approach may be most effective.

Some women suggested that the involvement of community groups and existing support networks would support effective dissemination of key messages. This supports previous studies where utilising existing community groups to initiate discussion and share information was an effective way of sharing key public health within culturally and linguistically diverse communities, $(18,19)$. In addition to engaging community groups, the importance of receiving consistent health messages from a trusted health professional was also identified in our study. Continuity of care, which can support and facilitate a trusting and respectful relationship, has been shown to have a positive impact on perinatal outcomes $(20,21)$.

A number of participants identified the need for visual as well as written materials and that many women utilise social media and online technology to access information. Mackintosh and others (2020), in their study of over 630 postnatal women in London, found that women used a range of online resources and apps to access advice and 'self-diagnose' potential complications in pregnancy (22). However other research has found that current tools are not necessarily reliable, there are for instance a number of online apps for reduced movements 
which do not provide evidence based advice (23). In previous work we have found that coproduced digital animation is acceptable and accessible way of communicating evidence based information in the perinatal period (24). Many women in this study thought the use of short videos, viewed through their phone would be effective in backing up the key messages around stillbirth prevention communicated by the midwife.

Our findings reveal the complexity of health professionals discussing stillbirth prevention with women from culturally and linguistically diverse backgrounds within the antenatal period and the importance of approaching the subject in a sensitive manner. However, as Warland and Glover (2015) argue, in order to successfully communicate these key messages, it is essential that health professionals have the resources and training to feel comfortable to talk to women from BAME backgrounds and their families about stillbirth and how to keep their babies safe (25). Focussed workshops to support maternity care providers to have these discussions have been shown to improve their knowledge and their intention to discuss stillbirth with women in their care in the future (26). In this context, such workshops would need an emphasis on developing cultural competence and addressing potential language barriers to ensure that the key messages are acceptable and accessible for BAME women. It is also worth noting that the women in this study originated from 18 different countries with heterogenous backgrounds and therefore when communicating key messages with BAME women, this heterogeneity must be recognised. What is considered acceptable and accessible for one BAME woman, may not be for another.

\section{Strengths and Limitations}

A key strength of this study was the engagement of women from a wide range of cultural backgrounds who had received maternity care from a range of Trusts. This helps to provide 
some insights into the variety and range of awareness and perceptions of stillbirth and associated public health messages. Despite this, our findings cannot be generalised to all women from BAME communities. Data collection was impacted by the COVID-19 pandemic and the planned focus groups were changed to individual interviews. We therefore lost the opportunity to exploit the advantages of focus groups in exploring perceptions of culturally and linguistically diverse groups (27) and utilising group dynamics to gain more depth to discussions (28). However, the required change to individual interviews may have allowed women who may have not spoken out in a focus group to explore their perceptions of stillbirth.

\section{Conclusion}

Our study found that there is still a low awareness of stillbirth and associated modifiable risk factors amongst women from BAME communities in the UK and that stillbirth as a term did not routinely exist in BAME women's narrative of perinatal health. This introduces an additional complexity for health professionals discussing stillbirth prevention with women from culturally and linguistically diverse backgrounds in the antenatal period and highlights the importance of approaching the subject in a sensitive manner. In order to address the persistent inequalities in stillbirth rates, it will be important to engage local communities and develop a range of co-produced, accessible resources to deliver effective public health messages. Messages need to be accessible and acceptable and they need be communicated effectively. As our study shows, who and how information is developed and shared is significant. Health professionals, in particular midwives who have developed a trusting relationship with the women will be key to ensuring the acceptability and accessibility of these messages. 
Ethics approval and consent to participate

- Ethical approval was obtained by University of Huddersfield ethics committee SREIC/2019/132. All participants gave (recorded) consent to take part. All methods were performed in accordance with the relevant guidelines and regulations

Consent for publication

$$
\text { Not applicable }
$$

Availability of data and materials

The dataset generated and analysed during the current study are not publicly available as they may contain information that could be confidential, but are available from the corresponding author on reasonable request.

\section{Competing interests}

The authors declare that they have no competing interests

Funding

- Funding was received from the University of Huddersfield internal funding scheme

Authors' contributions

- TS had overall responsibility of the study and secured funding. TS, MC and CK conceived of the idea, NA and MC collected the data. TS and MC undertook the analysis with input from NA. All authors were responsible for the drafting of the manuscript. All authors reviewed and gave approval for the final version of the manuscript.

Acknowledgements: We would like to thank the voluntary organisations for supporting the study

\section{References}

1. Flenady V, Wojcieszek AM, Middleton P, Ellwood D, Erwich JJ, Coory M, et al. Stillbirths: recall to action in high-income countries. The Lancet. 2016;387(10019):691-702.

2. Draper ES, Gallimore ID, Smith LK, Fenton AC, Kurinczuk JJ, Smith PW, et al. MBRRACE-UK Perinatal Mortality Surveillance Report UK Perinatal Deaths for Births from January to December 2018. The Infant Mortality and Morbidity Studies, Department of Health Sciences, University of Leicester; 2020.

3. Getahun D, Ananth CV, Kinzler WL. Risk factors for antepartum and intrapartum stillbirth: a population-based study. AJOG 2007;196(6):499-507. 
4. Rumbold AR, Yelland J, Stuart-Butler D, Forbes M, Due C, Boyle FM, et al. Stillbirth in Australia 3: Addressing stillbirth inequities in Australia: Steps towards a better future. Women and Birth. 2020;33(6):520-5.

5. $\quad$ Gordon A, Chan L, Andrews C, Ludski K, Mead J, Brezler L, et al. Stillbirth in Australia 4: Breaking the Silence: Amplifying Public Awareness of Stillbirth in Australia. Women and Birth. 2020;33(6):526-30.

6. Tennant PWG, Rankin J, Bell R. Maternal body mass index and the risk of fetal and infant death: a cohort study from the North of England. Human Reproduction. 2011;26(6):1501-11.

7. Flenady V, Koopmans L, Middleton P, Frøen JF, Smith GC, Gibbons K, et al. Major risk factors for stillbirth in high-income countries: a systematic review and meta-analysis. The Lancet. 2011;377(9774):1331-40.

8. Stacey T, Tennant P, McCowan L, Mitchell E, Budd J, Li M, et al. Gestational diabetes and the risk of late stillbirth: a case-control study from England, UK. BJOG 2019;126(8):973-82.

9. Heazell AEP, Budd J, Li M, Cronin R, Bradford B, McCowan LME, et al. Alterations in maternally perceived fetal movement and their association with late stillbirth: findings from the Midland and North of England stillbirth case-control study. BMJ Open. 2018;8(7):e020031.

10. Cronin RS, Li M, Thompson JMD, Gordon A, Raynes-Greenow CH, Heazell AEP, et al. An Individual Participant Data Meta-analysis of Maternal Going-to-Sleep Position, Interactions with Fetal Vulnerability, and the Risk of Late Stillbirth. EClinMed. 2019;10:49-57.

11. Heazell AEP, Timms K, Scott RE, Rockliffe L, Budd J, Li M, et al. Associations between consumption of coffee and caffeinated soft drinks and late stillbirth-Findings from the Midland and North of England stillbirth case-control study. Eur J Obstet Gynecol Reprod Biol. 2021;256:471-7.

12. Braun V, Clarke V. Using thematic analysis in psychology. Qualitative Research in Psychology. 2006;3(2):77-101.

13. Nuzum D, Meaney S, O'Donoghue K. The public awareness of stillbirth: an Irish population study. BJOG: An International Journal of Obstetrics \& Gynaecology. 2018;125(2):246-52.

14. Brierley-Jones L, Crawley R, Lomax S, Ayers S. Stillbirth and Stigma: The Spoiling and Repair of Multiple Social Identities. OMEGA - Journal of Death and Dying. 2014;70(2):143-68.

15. Pollock D, Ziaian T, Pearson E, Cooper M, Warland J. Understanding stillbirth stigma: A scoping literature review. Women and Birth. 2020;33(3):207-18.

16. Sather $M$, Fajon $A-V$, Zaentz R, Rubens CE, the GRG. Global report on preterm birth and stillbirth (5 of 7): advocacy barriers and opportunities. BMC Preg Child. 2010;10(1):S5.

17. Ahmed J, Raynes-Greenow C, Alam A. Traditional practices during pregnancy and birth, and perceptions of perinatal losses in women of rural Pakistan. Midwifery. 2020;91 doi.org/10.1016/j.midw.2020.102854

18. Escribà-Agüir V, Rodríguez-Gómez M, Ruiz-Pérez I. Effectiveness of patient-targeted interventions to promote cancer screening among ethnic minorities: A systematic review. Cancer Epidemiol. 2016;44:22-39.

19. Lu M, Moritz S, Lorenzetti D, Sykes L, Straus S, Quan H. A systematic review of interventions to increase breast and cervical cancer screening uptake among Asian women. BMC Public Health. 2012;12:413.

20. Sandall J, Soltani H, Gates S, Shennan A, Devane D. Midwife-led continuity models versus other models of care for childbearing women. Cochrane Database of Systematic Reviews. 2016(4). 
494 21. Rayment-Jones H, Harris J, Harden A, Khan Z, Sandall J. How do women with social risk 495 factors experience United Kingdom maternity care? A realist synthesis. Birth 2019;46(3):461-74.

496 22. Mackintosh N, Agarwal S, Adcock K, Armstrong N, Briley A, Patterson M, et al. Online 497 resources and apps to aid self-diagnosis and help seeking in the perinatal period: A descriptive 498 survey of women's experiences. Midwifery. 2020;90:102803.

499 23. Daly LM, Boyle FM, Gibbons K, Le H, Roberts J, Flenady V. Mobile applications providing 500 guidance about decreased fetal movement: Review and content analysis. Women and Birth.

501 2019;32(3):e289-e96.

502 24. Haith-Cooper M, Stacey T, Bailey F, Broadhead-Croft S. The Co-development and Feasibility503 Testing of an Innovative Digital Animation Intervention (DAISI) to Reduce the Risk of Maternal Sepsis 504 in the Postnatal Period. Matern Child Health J. 2020;24(7):837-44.

505 25. Warland J, Glover P. Talking to pregnant women about stillbirth. BMC Preg Child. 506 2015;15(Suppl 1):A12-A.

507 26. Warland J, Dorrian J, Pollock D, Foord C. 'InUTERO': The effectiveness of an educational half 508 day stillbirth awareness workshop for maternity care providers. Nur Educ Tod. 2020;85:104298.

509 27. Halcomb EJ, Gholizadeh L, DiGiacomo M, Phillips J, Davidson PM. Literature review:

510 considerations in undertaking focus group research with culturally and linguistically diverse groups. 511 Journal of Clinical Nursing. 2007;16(6):1000-11.

512 28. Robson C, McCartan K. Real World Research - Fourth Edition: Wiley; 2016. 


\section{Supplementary Files}

This is a list of supplementary files associated with this preprint. Click to download.

- Supplementarytable.docx 